\title{
EL HABITUS DE LA EDAD
}

PATRICIO RÍOS SEGOVIA*

\begin{abstract}
RESUMEN
Una teoría de la producción de las edades en las modulaciones de Pierre Bourdieu, sería un proyecto abortado sin una aproximación en profundidad a la noción del habitus de la edad. Operando con la noción de un meta código de la edad, el artículo se interna en el análisis de la articulación entre campo social de la edad, sus dos subcampos (longevidad y clases de edad) para distinguir, desde la realidad de la cultura dominante moderna, la producción social de habitus de edad diferenciados entre sí (niñez, juventud, adultez, adultez mayor, etc.), distinguiendo además el habitus generacional, vinculado al subcampo de las generaciones. El artículo postula los elementos conceptuales con los cuales está construido como herramientas útiles para la gerontología y los otros discursos que abordan las edades.
\end{abstract}

PALABRAS CLAVE: HABITUS DE LAS EDADES, HABITUS PROCESO HISTÓRICO, CAMPO SOCIAL DE LA EDAD.

* Investigador Corporación AÑos, Observatorio de la Vejez y del Envejecimiento en Chile. E-Mail: rioseg@live.cl. 


\title{
RESUMO
}

Uma teoria da produção das idades, nas modulações de Pierre Bourdieu, seria um projeto fracassado sem uma abordagem profunda da noção de habitus da idade. Operando com a noção de uma meta código da idade, o artigo aprofunda a análise da articulação entre campo social da idade, seus dois subcampos (longevidade e classes de idade) para distinguir, a partir da realidade da cultura moderna dominante, a produção social do habitus de idade em diferentes fases (infância, juventude, vida adulta, terceira idade, etc.), distinguindo, além disso, o habitus geracional, vinculado ao sub-campo das gerações. $\mathrm{O}$ artigo postula os elementos conceituais com os quais está construído como ferramentas úteis para a gerontologia e os outros discursos que abordam as idades.

\section{PALAVRAS CHAVE: HABITUS DAS IDADES, HABITUS PROCESSO HISTÓRICO, CAMPO SOCIAL DA IDADE}

\begin{abstract}
A theory of age in modular production by Pierre Bourdieu, would be a rejected project without even being close in depth to the notion of habitus of age. Working on the notion toward a goal of code of age, this article analyses the articulation between social field and age. It's two sub-fields (longevity and classes) distinguish the reality of the modern dominant culture, social production of habitus of age differentiated from (childhood, youth, adulthood, the elderly, etc.). It also distinguishes generational habitus, in relation to the sub-fields of these generations. This article applies for conceptual elements which are constructed as useful tools for gerentology and other topics which address age.
\end{abstract}

KEY WORDS: HABITUS OF AGE, HISTORIC HABITUS, SOCIAL FIELD OF AGE 


\section{INTRODUCCIÓN}

LA EDAD ES UN campo social en el sentido que el sociólogo francés Pierre Bourdieu lo ha definido. Estructurado por los subcampos sociales de la longevidad, de las clases de edad y de las generaciones, el campo de la edad se constituye en las disputas de agentes diversos por un capital simbólico que está constituido también por la edad (Gutiérrez y Ríos, 2006). Desde una perspectiva gerontológica, los habitantes del campo de la edad somos los envejecientes, es decir, sujetos de cualquier edad, desde el/la no nato/a hasta el/la de más años. Descrito el campo social de la edad en sus lineamientos más gruesos en el artículo citado de Gutiérrez y Ríos, corresponde ahora dar cuenta del habitus de la edad. Es la intención y el objetivo de las líneas que continúan.

\section{EL HABITUS EN PERSPECTIVA: OBJETIVIDAD/SUBJETIVIDAD/PRAXIS}

\section{a) El habitus y el habitante habitado}

Desde una cierta dimensión provocadora de la filosofía de Bourdieu, los sujetos somos habitantes, instalaciones vivas, con la capacidad de desplazarnos desde posiciones de dominancia a las de subordinación, y viceversa, al interior de reductos diferenciados que Bourdieu llama campos sociales. Cada sujeto es, pues, un individuo situado, y en su terminología, un agente instalado en campos diferenciados del espacio social y de la historia.

La visión de Bourdieu descansa por una parte, en la capacidad del conocimiento sociológico de develar los campos, las realidades objetivas, que componen el espacio social de un momento dado de la historia de una comunidad $\mathrm{y}$, porque ello es posible, simultáneamente, proponer una herramienta conceptual para recrear en el habitante, en el situado, la habitación que habita, vale decir las condiciones objetivas que internalizó, procesadas como esquemas, organizaciones de la percepción o disposiciones, es decir, procesadas en cuanto habitus.

Si el espacio social - poblado por los diferentes campos sociales - acompañan a los envejecientes desde antes del alumbramiento constituyendo su hábitat, sus circunstancias históricamente fundadas; los habitus representan la otra cara de la moneda, la organización subjetiva de la interacción con lo objetivo, lo que siendo el resultado de 
las prácticas es a la vez un sentido en sí, una lógica de la práctica que ha emergido de este chocar del habitante con lo que habita y que, a su vez, en lo que es su historia, también ayuda a transformar.

El habitus, no es así, un mero reflejo especular de lo objetivo en lo subjetivo, sino una nueva síntesis de lo objetivo que el habitante de lo objetivo incorpora, haciéndolo cuerpo.

La síntesis que representa el concepto de habitus entre objetividad y subjetividad es de una naturaleza particular y puede describirse en relación a referentes diferentes:

i) En relación al mundo práctico: el habitus, en cuanto incorporación ya hecha por el sujeto a lo largo de su existencia del mundo objetivo constituye «un sistema de disposiciones duraderas predispuestas para funcionar como estructuras estructurantes, es decir, en tanto que principio de generación y estructuración de prácticas y representaciones que pueden estar objetivamente reguladas y ser regulares sin constituir el producto de la obediencia a reglas». A la manera de la racionalidad estructuralista que tiende a poner el énfasis en concebir lo objetivo como conjunto de códigos y reglado de lo objetivo, el habitus, incorporación de las constantes del mundo objetivo en el cual ha participado el sujeto, no es la interiorización de códigos, de conjuntos de reglas. No es una construcción ni una reconstrucción lógica del mundo objetivo (mundo encriptado). No obstante, los habitus son «estructuras» (incorporación de lo ya hecho en el mundo práctico) «estructurantes» (usables por el sujeto en empleos nuevos en el mundo práctico). Con ello, el habitus, expresa una nueva síntesis conceptual en virtud de la cual, el mundo externo es sujeto y el sujeto es «agente» en el mundo externo, expresando las dos dimensiones presentes en las prácticas, a saber, una ritual (mecánica, previsible, regular y regulada) y otra estratégica (libre, original).

ii) Ligado a una complejidad temporal: el habitus vincula varios tiempos. El primero de ellos refiere a su carácter de realidad en el sujeto, a su función en el sujeto que no es otra que la de servir de orientación del mismo en la vida práctica, en su vida cotidiana. Y en esta dinámica, las cosas suceden en la urgencia del transcurrir del tiempo: ni antes ni después, sino en el momento que es preciso. Además, de manera adecuada. El habitus conduce al sujeto en estas exigencias de la vida práctica y en ello sirve de puente entre el sujeto y la inmediatez del tiempo. 
Por otra parte, por su origen, el habitus se constituye en los sujetos agentes en la práctica social, al interior de campos sociales concretos, generados históricamente. Por lo mismo, al actualizarse en la vida cotidiana de los sujetos, «el habitus produce prácticas [...] conformes a los esquemas engendrados por la historia» (Bourdieu, 1980:91), «Historia incorporada, hecha naturaleza, y por ello olvidada en cuanto tal, el habitus es la presencia actuante de todo el pasado del que es producto: de partida, es el que confiere a las prácticas su independencia relativa en relación a las determinaciones exteriores del presente inmediato» (Bourdieu, 1980:94).

El tercer componente temporal con el cual conecta el habitus es una de las características que lo asemeja al juego, al «sentido del juego».

Constituido en la práctica social, en la incorporación en la subjetividad del sujeto de las condiciones materiales del campo en el cual el sujeto es agente, el habitus orienta con propiedad las conductas del sujeto, lo dota de «esa especie de sentido práctico de lo que hay que hacer en una situación determinada - lo que en deporte se llama sentido del juego, arte de anticipar el desarrollo futuro del juego que está escrito en punteado en el estado presente del juego- $\longrightarrow$ (Bourdieu, 1997:40).

El habitus está en condiciones, pues, de prever situaciones que le permitan al sujeto sacar ventajas de su juego. La nueva síntesis de lo objetivo que es el habitus, como se ve, posee una triple relación con tiempos diferentes.

iii) Vinculado a la categoría cultural de lo razonable y al concepto de racionalidad práctica: el habitus es un tipo de conocimiento que está tan lejos de lo racional como de lo irracional, o de lo inconsciente. Así lo expresa Bourdieu: «Los esquemas del habitus, formas de clasificación originarias, deben su eficacia propia al hecho de que funcionan más allá de la conciencia y del discurso, luego fuera de las influencias del examen y del control voluntario» (Bourdieu, 1988:477). Su eficacia consiste en generar conductas que sean adecuadas, efecto este último de lo que es presentado como «sentido práctico» o como «sentido del juego», cuestión del todo coherente con la concepción central de los campos, espacios sociales donde los agentes luchan o apuestan por la conservación o el aumento del capital específico que el campo ofrece. En ello, el habitus proporciona las orientaciones centrales y suficientes como para producir actuaciones razonables o adecuadas. Tal cual puede apreciarse, la noción de habitus está indisolublemente ligada a la vida práctica, a la vida cotidiana. Es el depósito de racionalidad práctica. 


\section{b) Habitus, habitus de clase y habitus de edad}

El proceso de in/corporación del habitus implica dos operaciones centrales en el pensamiento de Bourdieu: i) la introyección de las condiciones materiales concretas en las que el individuo se desenvuelve por su posición social: acceso, variedad y modalidad de consumo de bienes sociales y de mercado, estructura y clima de relaciones sociales, frecuentación o lejanía de bienes culturales. ii) La inculcación y apropiación por parte del sujeto de un arbitrario cultural, facturado tanto desde la familia y la escuela, como desde el entorno.

La inculcación/apropiación del arbitrario cultural opera a través de acciones pedagógicas espontáneas - familia, medios de comunicación, comunidad de pares- $-\mathrm{y}$ a través de acciones pedagógicas racionales - escuela, influencias paternas y maternas intencionadas, transmisión consciente de contenidos de vida por parte de hermanos mayores, parientes-. Producto de estas intervenciones, los condicionamientos objetivos — de posición social - se instalan en la forma de esquemas y disposiciones al interior de los sujetos.

Se sigue de esto que los sujetos que experimentan condiciones de existencia similares tenderán a compartir sistemas de disposiciones análogos, es decir, habitus análogos. Tales habitus son los habitus de clase. ${ }^{1}$

1 Con el fin de ser rigurosos en este punto se hace necesario aludir en primer lugar al concepto de «clase social» de Bourdieu y luego a lo que él entiende por «habitus de clase». i) en relación a la categoría de la clase social, Bourdieu, apartándose de Marx, establece una división tajante entre «la clase social» movilizada, sustancializada, introducida por Marx y la clase «en el papel», categoría del método sociológico: «Esta clase 'en el papel' tiene la existencia teórica propia de las teorías: en la medida en que es el producto de una clasificación explicativa, del todo análoga a la de los zoólogos o los botánicos, permite explicar y prever las prácticas y las propiedades de las cosas clasificadas $\mathrm{y}$, entre otras cosas, las conductas de las reuniones grupales. No es en realidad una clase, una clase actual, en el sentido de grupo y de grupo movilizado para la lucha; en rigor podríamos hablar de clase probable, en tanto conjunto de agentes que opondrá menos obstáculos objetivos a las empresas de movilización que cualquier otro conjunto de agentes» (Bourdieu, 1990:282); ii) en cuanto al habitus de la clase social, Bourdieu vincula el proceso de constitución de las estructuras subjetivas de los sujetos (habitus) a la soldadura compleja entre condiciones objetivas semejantes compartidas por sujetos específicos y constitución de estructuras 
El habitus de la clase social es el primero en instalarse. Corresponde a las adquisiciones que se hacen en la primera infancia y en la infancia como correlato de las condiciones materiales en las cuales se existe. ${ }^{2}$ La formación del habitus de edad es parte de este proceso y se da al interior de una dinámica que no ha sido suficientemente atendida. A continuación se abren direcciones de trabajo que pudieran explicar la emergencia y la constitución diferenciada del habitus de la edad.

\section{CONSTRUCCIÓN DEL HABITUS DE LA EDAD}

\section{a) Mundo animal y edad}

Desde un punto de vista estrictamente biológico, y de especie, los mamíferos obedecen al mandato gregario de la supervivencia, lo que implica, una dinámica que equilibre, por una parte, desempeños individuales al interior de un colectivo - manada, tribu, jauría - y, por otra parte, desarrollo orgánico de los individuos, vale decir, tiempo, edad. En su estar en el grupo, los individuos, para sobrevivir, pasan desde una condición de dependencia absoluta de los progenitores hasta otra de autonomía de los mismos, ciclo que se perpetúa en el cumplimiento de la renovación de la especie por medio de la reproducción sexual. El esquema 1 ilustra lo que se viene afirmando.

subjetivas semejantes en los sujetos considerados, estructuras que a su vez se transforman en estructurantes. En palabras de Bourdieu «Sistema de esquemas generadores de prácticas que expresa de forma sistémica la necesidad y las libertades inherentes a la condición de clase y la diferencia constitutiva de la posición, el habitus aprehende las diferencias de condición, que retiene bajo la forma de diferencias entre unas prácticas enclasadas y enclasantes (como productos del habitus), según unos principios de diferenciación que, al ser a su vez producto de estas diferencias, son objetivamente atribuidos a éstas y tienden por consiguiente a percibirlas como naturales» (Bourdieu, 1988:170-171).

2 Hay que recordar que los habitus se especifican en función de las divisiones en campos sociales de la realidad objetiva. «Sin embargo, detrás de la multiplicidad se esconden los principios generadores integradores de las clases sociales ya que el habitus de clase funciona como la forma incorporada de la condición de clase y de los condicionamientos que ella impone» (Degl'Innocenti, S/F). 


\section{ESQUEMA 1}

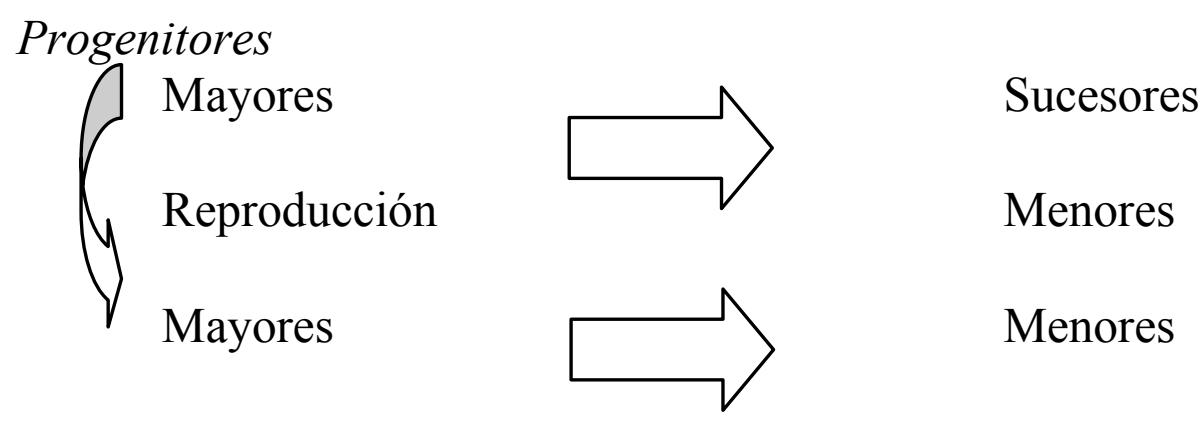

Varios progenitores y diversos sucesores coexistiendo en un mismo tiempo espacio denotan la existencia de una unidad social funcionando y por lo mismo de jerarquías, de dinámicas de cooperación y de competencia, y de mecanismos básicos de control. En otras palabras, se enfrenta una unidad social como una jauría o una manada o una tribu de animales. El esquema 2 ubica parte de estas afirmaciones.

\section{ESQUEMA 2}

Progenitores

Mayores

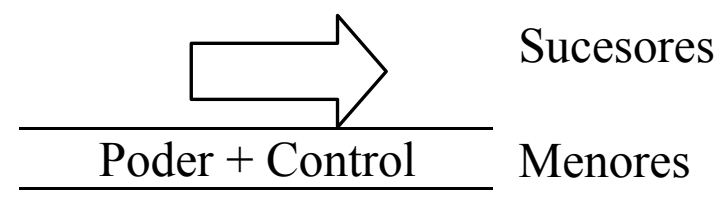

Respecto de la figura anterior se ha recogido una sola observación: la ordenación jerárquica, la dinámica del poder que dispone las piezas de grandes a chicos, de controladores a controlados, mecanismo en el que es necesario detenerse porque, entre otras razones, apunta a una estructura elemental del funcionamiento social: la estructura elemental de la edad. Dentro del mundo animal, la referencia a la cual se recurre aquí, es la de la organización social observada entre monos, felinos y cánidos, es decir, de animales pertenecientes a los mamíferos superiores.

Mundo animal (mamíferos: monos, felinos, cánidos)

Roles sociales y desarrollo orgánico

Dentro de estas clases de animales, la ordenación etaria se manifiesta, por ejemplo en: 
i) Las conductas conocidas como parentales, construidas sobre la base de la relación de dependencia de los menores y su progenitora.

ii) Las conductas de juego, construidas entre los miembros de la familia, jauría o manada sobre la base de relaciones horizontales, entre pares, aunque también es posible detectarlas entre mayores con menores.

iii) Las conductas de cooperación entre mayores para conseguir el alimento, en ocasiones acompañados por menores.

iv) Las conductas de defensa del territorio, enfrentamientos protagonizados por mayores.

v) Las luchas de sucesión de puestos de poder en la jerarquía organizacional que enfrentan a mayores jóvenes con mayores menos jóvenes.

vi) Las luchas por el apareamiento que ponen en conflicto a mayores más o menos jóvenes también (Estramil, 2007).

No es del caso extender esta incursión por la etología animal y lo que se ha dicho es suficiente para enfatizar el hecho de que en el orden animal se observa que la maduración o desarrollo orgánico (energía y envergaduras físicas), junto con el sexo, son principios suficientes de ordenación social al posibilitar la adscripción de los individuos a uno u otro rol excluyéndolos de otros.

En la tabla siguiente se listan algunos roles sociales observados en parte del mundo de los mamíferos adscribiéndolos a una u otra dimensión del desarrollo orgánico de los individuos. Este último se concibe dividido en dos categorías: mayores/menores y en dos subcategorías: excluyente/no excluyente. El resultado se presenta en la siguiente tabla.

\begin{tabular}{|l|c|c|c|c|}
\hline Roles sociales & $\begin{array}{c}\text { Mayor } \\
\text { Excluyente }\end{array}$ & $\begin{array}{c}\text { Mayor no } \\
\text { excluyente }\end{array}$ & $\begin{array}{c}\text { Menor } \\
\text { excluyente }\end{array}$ & $\begin{array}{c}\text { Menor no } \\
\text { excluyente }\end{array}$ \\
\hline $\begin{array}{l}\text { Autoridad } \\
\text { progenitora }\end{array}$ & $\mathrm{X}$ & - & - & - \\
\hline $\begin{array}{l}\text { Bajo tutoría proge- } \\
\text { nitora }\end{array}$ & - & - & $\mathrm{X}$ & - \\
\hline $\begin{array}{l}\text { Jefatura territorial } \\
\text { o de parentesco }\end{array}$ & $\mathrm{X}$ & - & - & - \\
\hline Luchas jefaturas & $\mathrm{X}$ & - & - & - \\
\hline Proveedor/a & - & $\mathrm{X}$ & - & $\mathrm{X}$ \\
\hline Luchas territoriales & $\mathrm{X}$ & - & - & - \\
\hline $\begin{array}{l}\text { Luchas } \\
\text { apareamiento }\end{array}$ & $\mathrm{X}$ & - & - & - \\
\hline $\begin{array}{l}\text { Participante } \\
\text { de juegos }\end{array}$ & - & $\mathrm{X}$ & - & $\mathrm{X}$ \\
\hline
\end{tabular}


Asomarse al mundo animal para preguntarse por las habilitaciones/inhabilitaciones en el orden social que trae aparejado el envejecimiento animal (o el desarrollo orgánico animal) ha sido útil en la medida que permite develar un código elemental de funcionamiento social vinculado al proceso de maduración orgánica o de envejecimiento.

Tal código se expresa en la tabla siguiente, en donde los referentes de código están al lado izquierdo mientras que los elementos situados a la derecha sólo dan origen a variantes: mayores (excluyentes/no excluyentes); menores (excluyentes/no excluyentes).

\begin{tabular}{l|l} 
Mayores & Excluyentes \\
\hline Menores & No excluyentes
\end{tabular}

Este universo, pues, se desenvuelve al interior de un mundo perceptivo codificado, pero no discreto, y lo que el código distingue son magnitudes. Los roles sociales, comparados con la sociedad humana, forman inventarios cerrados en los que importa que en determinadas funciones nunca aparecerá un menor (defensa territorial; lucha por apareo) y en otras, nunca un mayor (bajo tutoría); en cambio habrá otras - actividades de caza tutelada, por ejemplo - en las que podrá haber una mezcla entre mayores y menores; pero, bajo la tutoría (la invitación, la motivación) de la progenitora. Hay, en este funcionamiento social, una especie de acuerdo custodiado de la naturaleza: «si se está en actividades de lucha es porque ya se es mayor»; «si se caza es porque se es mayor». Qué tanto mayor o qué tanto menor, no hace diferencias. Se opera con grandes discontinuidades. Se instalan períodos de dependencia más o menos largos entre el/la más pequeño/a y los progenitores que se relajarán con el correr del tiempo, hasta diluirse en las distintas formas de la autonomía.

En este tipo de contexto, un individuo se encuentra permanentemente rodeado de otros, interactuando con otros. En esa misma medida, los individuos, en la práctica, tendrán que aprender, entre otras señales, a reconocer las relaciones que les permite interactuar con los otros, y en ese proceso, a conocer a los otros, en sus múltiples dimensiones, también en la de aquella de quienes ostentan mayor o menor desarrollo orgánico. Frente a los mayores y a los menores, la práctica de convivencia a lo largo del tiempo, ubicará al individuo en las distintas relaciones posibles con unos y con otros: primero, habrá dependencia, más adelante subordinación y más adelante, en el mundo de pa- 
res $^{3}$, la competencia y la colaboración. Como mayor, reconocerá la sumisión de los menores.

Sin sistemas simbólicos desde y con los cuales interactuar, esta orientación de «mayores» o «menores» con la que los individuos se conducen en la vida práctica de la manada, jauría o tribu, es una indicación que se deposita en el individuo por la vía de una práctica societal apoyada en el desarrollo orgánico de mecanismos habilitantes de los individuos. Esta indicación morirá con el individuo, el que, por otra parte, cumplirá con su ciclo, toda vez que haya logrado aportar sucesores - desde su condición de macho o hembra - para la continuidad de la especie.

\section{b) La edad y el código de la meta edad}

A diferencia del mundo animal, en las sociedades humanas se opera con categorías culturales y el mayor o menor grado de desarrollo orgánico de los sujetos se expresa en la categoría cultural de la edad. Los sistemas culturales se encargan de convertir estas magnitudes de lo mayor y de lo menor que operan en la práctica animal, en unidades discretas mediante la aplicación de artefactos complejos de medición del tiempo y de otros mecanismos de sofisticación creciente como las modernas estrategias de control social.

De esta manera, teóricamente, la edad no es más que una cantidad. Esta cantidad, despojada de las significaciones culturales, en el puro nivel analítico y ahistórico, está constituida, a diferencia del código perceptivo animal, por categorías perceptivas estructuradas en el lenguaje en un sistema con tres componentes: «mayor/menor/ni mayor ni menor»».

Los componentes de este meta código se instalan en los habitus de los individuos en el proceso histórico de existir, como categorías de identidad, producto de prácticas sociales específicas en que se ven envueltos los individuos y que abarcan desde los sistemas complejos de medir el tiempo - los calendarios- hasta las construcciones más apegadas a los desarrollos históricos, al juego de dominantes y dominados, como son las clases de edad.

De esta manera, un código que es mera percepción en el mundo animal, se constituye en código cultural, producto del efecto simultáneo de la existencia en cada período histórico de estructuraciones

3 Conocido en la práctica en la vida compartida con los hermanos. 
complejas como los campos sociales - incluido el campo de la edady de la introyección de esos campos sociales por parte de los individuos en la realidad del habitus. De este modo, al interior de las especificidades del habitus - habitus de clase social, de oficio- el habitus de la edad se constituye en la práctica social del individuo de habitante del campo de la edad en dos subcampos:

i) El subcampo de la longevidad, en el cual, los componentes del código de la meta edad se especifican en el habitus de la edad a la manera de distancias que separan el momento del estar del momento del nacimiento del individuos, permitiendo con ello, la operatividad de los distintos componentes del meta código: quienes se encuentran a una misma o parecida distancia pertenecerán a la categoría «ni mayores ni menores», quienes se encuentren a una mayor distancia porque han nacido antes, integrarán la categoría de los «mayores» y quienes se encuentren a otra distancia, porque han nacido después, constituirán los menores.

Por otra parte, la experiencia de la «durabilidad» en el tiempo con la cual se vive cada época (la longevidad) es computada también por el habitus de la edad, dato que orientará o influirá las prácticas sociales de los individuos: «tenía toda una vida por delante» (longevidad menor de la esperada)/«tenía ya sus años» (longevidad esperada).

ii) El subcampo de las clases de edad. El habitus de la edad introyecta aquí en los individuos el ordenamiento y distribución que la sociedad realiza de los individuos en función de sus edades calendario adosadas a un conjunto de prohibiciones y de permisividades sociales y culturales reconocidas y legitimadas por los mecanismos de la dominación de clase.

De esta manera, cada época histórica escribirá su propia manera de encarnar el código de la meta edad en campos y subcampos de la edad específicos los que serán asumidos por cada individuo a través del habitus de edad de la época respectiva.

El diagrama permite visualizar de qué manera, dos períodos históricos alejados en el tiempo, (sociedad de la Edad Media y sociedad moderna) asumen el meta código de la edad en campos y en subcampos sociales de la edad diferentes.

En el primer caso, en el período histórico de la Edad Media, el parámetro para la distinción de edades diferentes era de treinta años. La longevidad de la época equivalía a poco más de un tercio de la 
actual. Por otra parte, las circunstancias materiales en las cuales sucedía la existencia de los individuos era de una gran vulnerabilidad. Las epidemias, la falta de respuestas médicas a las enfermedades, los períodos de hambruna, la desprotección frente a la violencia, implicaban tasas de mortalidad elevadas, en especial entre niños y mujeres. Entre estos dos datos estructurales se constituía el habitus de edad que sustentaba el funcionamiento social. En este último, los menores eran adscritos a faenas de mayores en cuanto fuera posible, como aprendices en los talleres industriales o como trabajadores del campo. De este modo, los menores eran subsumidos por la clase de edad de la adultez de los sujetos, al igual que la juventud, invisibilizada.

La realidad de las sociedades modernas es distinta. Puede apreciarse que en el campo de la edad de estas sociedades, el componente «menor» del meta código está mediatizado por las clases de edad infancia/adolescencia/juventud y el de «mayor» por las clases de edad «adulto», «adulto mayor». A su vez, el subcampo de la longevidad anota las cifras más altas de esperanza de vida hasta ahora conocidas. El habitus de edad de los sujetos en estas sociedades es, pues, distinto. Las prácticas sociales generadas por clases de edad diversificadas, introyectan habitus de edad en los sujetos que les permite reconocerse en ellas como una parte de sus respectivas identidades. 


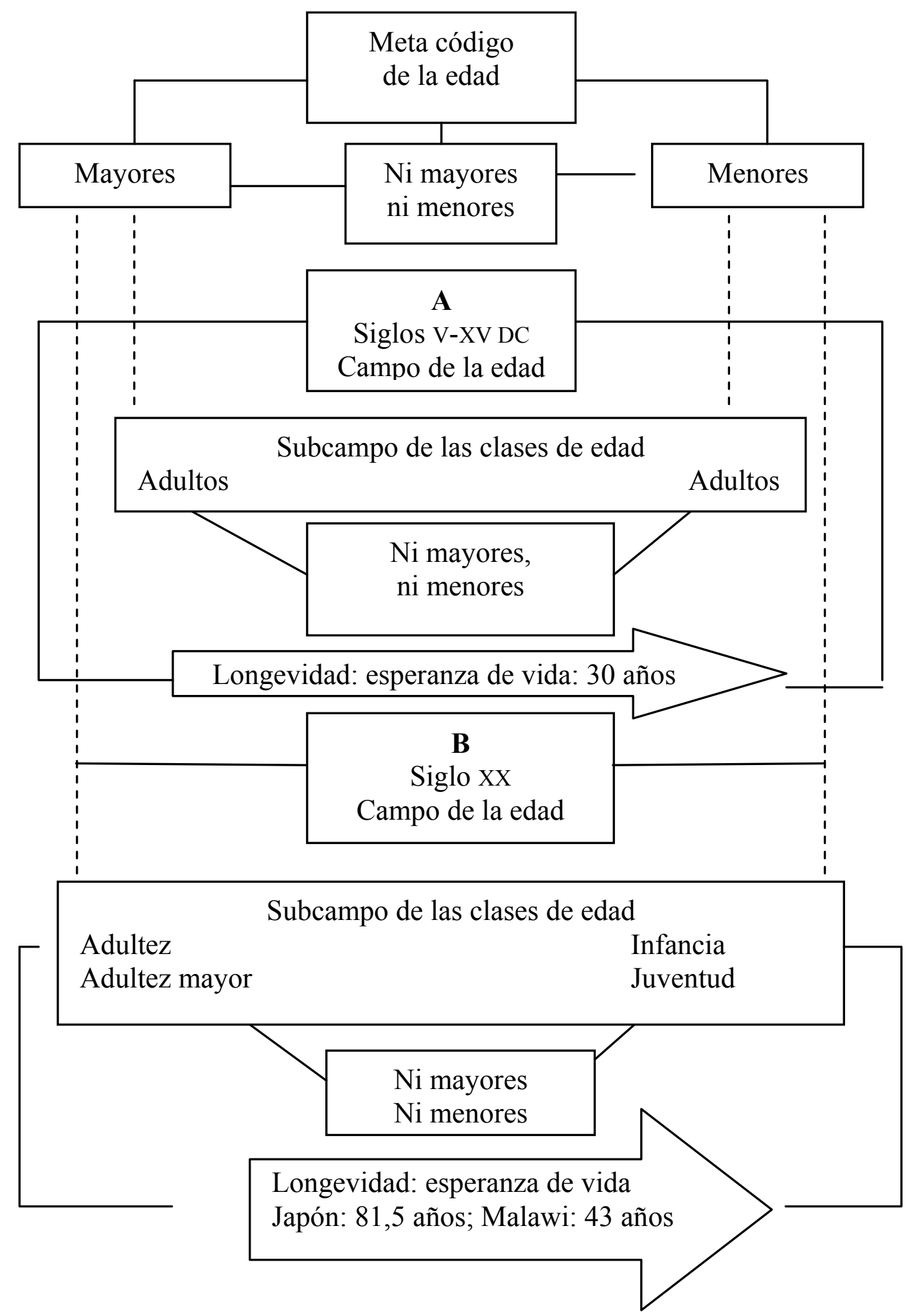




\section{EL HABITUS Y LAS CLASES DE EDAD}

Las primeras cristalizaciones históricas del campo de la edad corresponden a las clases de edad. Estas últimas enclasan a los sujetos, los clasifican, en agentes marcados, diferentes, tanto por sus edades biológicas como por sus edades sociales.

Así, cada sujeto introyecta sus habitus de edad en la medida en que participa en los procesos históricos de construcción de las clases de edad. En estos últimos es observable dinámicas sociales en las que los adultos ordenan el funcionamiento social de acuerdo a las divisiones culturales de las edades que distinguen las clases dominantes de una cultura dada en un período histórico dado.

En el subcampo de las clases de edad, los agentes que intervienen con mayor fuerza en su establecimiento forman parte del estamento burocrático. Es el Estado el que de manera legitimada tanto política como socialmente, en especial a través de su ordenamiento jurídico y educacional, implanta las estructuras que habrán de informar los habitus de las distintas clases de edad.

\section{a) El habitus de la infancia}

Prácticas del cuidado de la salud estatuidas por el sistema de salud así como las prácticas del sistema formal de educación y del ordenamiento jurídico están en la base del establecimiento del habitus de edad.

La niñez, supervigilada por la adultez, ha de cumplir con una serie de rituales de cuidado de la salud, lo que obliga a los adultos a capacitarse en los calendarios de vacunas, alimentación de los niños y otras prácticas estrictamente establecidas en función de edades, so pena de poner en riesgo el desarrollo infantil y con ello la construcción de uno de los capitales esenciales - la salud - en el desempeño social del adulto en formación.

Por otra parte, las prescripciones etarias del sistema escolar son estrictas en términos del inicio de ciclo básico y, a partir de ello, el orden etario de los 12 años de educación obligatoria se enriela en una lógica que uniformará a la población, la que deberá haber cumplido con la totalidad del ciclo entre los 6 y los 18 años de edad.

Con ello, la construcción de un habitus de edad en los sujetos adquiere un referente en términos de la producción de las clases de edad, porque las prácticas del funcionamiento social, direccionadas por los adultos, habrán aislado a un segmento de la población, por su edad, 
asignándole un destino específico, con funciones diferentes de otros segmentos etarios y prácticas que también les son propias, ayudando a visibilizarlas en cuanto clases de edad particulares. El efecto más poderoso de estas dinámicas consiste en la implantación de polos de interacción entre pares como un componente central de la construcción del habitus de la edad y de la identidad. Desde la familia, los menores (los niños) son institucionalizados cada vez de manera más temprana, en centros con otros menores, entre los cuales se construye básicamente la experiencia del par, (del «ni mayor ni menor») que habrá de ser contrastada con la experiencia de los mayores y de los menores, dentro de esos mismo centros, y, por cierto en el resto de los horizontes de las prácticas sociales en las que participa el sujeto. ${ }^{4}$

Por cierto, el escenario descrito de construcción del habitus de edad de la infancia y de la primera juventud obedece al escenario de las posiciones dominantes de la sociedad. Las condiciones materiales de existencia de los individuos enclasados socialmente contribuirá a diferenciar el habitus de la edad de los sujetos, con lo que será más riguroso hablar de infancias y de juventudes tempranas, así, en plural, en la medida en que el habitus de la edad se vive al interior del habitus de los dominados o de los dominantes.

Hay que insistir en este punto: el habitus de edad de la infancia corresponderá a la interiorización de las condiciones materiales concretas en las cuales transcurrieron los primeros años de la existencia. En ellas pudo o no existir la escuela o existir una escuela precaria o bien dotada o una escuela geográficamente distante del hogar con posibilidades. En una sociedad de clases, estas condiciones pueden ser muy adversas si se pertenece al grupo de los dominados desposeídos, como, por ejemplo, la inexistencia de la escuela por restricción drástica del capital económico familiar. En esta circunstancia o en otras acarreadas por la pobreza o la indigencia que no es del caso pormenorizar, el habitus de infancia del sujeto será muy diferente al introyectado en el sujeto de la clase dominante.

Si la escuela o la comunidad de pares es determinante en la formación del habitus de edad, también lo es la familia.

4 Esta operación de unión de funciones sociales específicas a edades específicas, como, por ejemplo, niñez=estudio/formación/escuela, obedece a una racionalidad social históricamente construida. Hasta el siglo XVIII, los niños no eran sujetos de educación ni la escuela una institución instalada. 
La primera versión de adultos que conoce el niño son sus padres. El adulto padre-madre y el adulto maestro/maestra de la escuela van introyectando en sus habitus y en la medida que su desarrollo lo permita, las primeras impresiones de lo que ser grande puede significar. En el hogar y/o en la escuela, con sus pares y con los adultos, el niño va incorporando primero las experiencias de toparse con los más grandes - los adultos- y también con los menos grandes que los adultos, pero más grandes que él mismo. Por último incorporará la experiencia de toparse con sus iguales, sus pares.

Puede apreciarse que estos contactos de los primeros años de vida son esenciales para la formación del habitus de la edad. Puesto en el mundo de los más chicos, el niño interioriza no sólo lo que los adultos predican sobre lo que significa ser niño, sobre las maravillas y sobre las desgracias de su condición, sino que también siente las coerciones de las cuales es objeto, así como las libertades de las cuales puede gozar. Siente las diferencias que tales coerciones o libertades pueden llegar a tener si se las experimenta con los pares, los más grandes o los adultos.

En este haz de relaciones etarias en que transcurren sus prácticas de niño, en su habitus de edad se van fijando las características estructurales, las invariables de la condición de ser niño que serán los rasgos distintivos de esta clase de edad, vale decir, el conjunto de permisividades y de prohibiciones con las que tropieza en el encuentro práctico de las edades del espacio cotidiano.

«No juegues con fuego», «no metas los dedos al enchufe», «no saltes de esa altura, eso es para los más grandes», «no me gustan los niños llorones», «no comas con la mano», «no dejes la ropa botada», «no saludes ni hables con los desconocidos en la calle», las prohibiciones.

«Sí, claro, puedes ir al cine», «bueno, llega más tarde», «está bien, anda a jugar un rato», "¿ésta era la figura que te faltaba? (en un álbum)», las permisividades.

«¿Y cómo es que $\mathrm{X}$ (un amigo, un hermano/a, algo mayor, pero niño) se come toda la comida?», «X (otro niño) siempre le obedece a su mamá, es un niño muy bonito», «a los niños que tienen pataletas les da hipo», los modelos.

Los componentes de una cierta pedagogía de la edad ejercida por los adultos: padres/profesores/hermanos/as mayores/parientes, inculcando la pertenencia a una cierta clase de edad.

La escuela y la familia además de la experiencia con los pares, terminan por formar el habitus de una clase de edad en los sujetos. Ello 
facilita que el niño, cuando llegue a adulto reproduzca con sus hijos/as, sobrinos/as esa clase de edad que su habitus atesora, o bien, que, habiendo cambiado las condiciones objetivas de la educación de los hijos, y habiendo identificado capitales nuevos destinados a mejorar su posición social relativa, modifique el habitus de su propia infancia y luche por hacer que su descendencia se haga de esos nuevos capitales.

\section{b) El habitus de la primera juventud}

A medida que la edad biológica aumenta, la importancia del grupo de pares en la transición del habitus de la clase de edad de la infancia hacia otro diferente - el de la clase de edad juvenil— también aumenta. Pero no sólo eso. Los adultos asimismo empujan hacia la construcción de una identidad etaria diferente a la del niño.

No adulto todavía y en transición del habitus de la niñez, este habitante del campo social de la edad - este envejeciente- se encuentra tironeado por su habitus de la niñez - que lo ancla en la sumisión del respeto de la autoridad de los adultos, especialmente bajo las figuras del padre, la madre, el/la profesor/a, los adultos que por alguna razón admira - y las fuerzas centrífugas que lo impulsan en una dirección contraria. Estas últimas están conformadas, por una parte, por las condiciones materiales de vida en las cuales existe y la posición social de los mayores que conforman su círculo más inmediato. Por otra parte, el intercambio con sus pares.

De hecho, el habitus de la nueva clase de edad a la cual se encamina debe tomar forma en una situación estructural de las relaciones entre las clases de edad diferente de aquella en la cual se conforma el habitus de la niñez. Lo que ha cambiado es el conocimiento, la presencia marcada de «lo menor». En la práctica, comienza a frecuentar a «los/las más chicos/as». Su manera de experimentar la edad ha cambiado: hasta algún momento sólo existe una vinculación con «lo mayor»; en algún otro punto de la trayectoria vital, comienza a experimentar también, lo menor.

El habitus de la clase de edad que sigue a la infancia se forma en la relación práctica de los tres componentes del meta código de la edad: «los menores»/«los mayores»/«los pares», a diferencia del habitus de la clase de la infancia que se constituye, básicamente, en la interacción con dos de los componentes del meta código de la edad: «los mayores»//《los pares». 
Esta diferencia hace que el habitus de la edad juvenil tenga más riqueza, mayor complejidad, en especial, porque los ejes de las relaciones dejan de ser sólo el de la sumisión (frente a los más grandes) y el de horizontalidad (frente a sus pares) para comenzar a experimentar también el de la dominación (con los menores).

Complejidad que aumenta también con la evolución del mundo de sus pares. Hasta algún momento, su relación con ellos no se reduce sólo a una horizontalidad no adjetivada, a una horizontalidad simétrica cuya identidad emerge sólo en su contraste con lo mayor. En algún momento, aparece una horizontalidad sexuada. Los pares ahora son una categoría compuesta por las distinciones «ni mayor que yo» + «ni menor que yo» + «hombre»/《mujer».

Las prohibiciones y las permisividades de las clases de edad se interiorizan ahora a partir de condiciones objetivas diferentes a las anteriores. Se interiorizan en unas prácticas que implican recibir órdenes, pero, a su vez, en las de dar órdenes; también en las de discutir órdenes. Todo ello dentro de una legitimidad asentada.

Mediante el acatamiento de las órdenes de los mayores - de los adultos - se afirma, se afiata y se reproduce el ordenamiento heredado de las relaciones de las clases de edad. Mediante el acto de impartir órdenes a los menores - por parte de los no adultos, pero más grandes-, se complejiza la condición de «niño», de su clase de edad. Se comienza a interiorizar la práctica de dominar a otro y por esa vía la noción de la dominación en las relaciones sociales.

\section{c) El habitus de la temprana juventud}

Crecer, ganar en años, envejecer, va de la mano con el cambio de mirada de los adultos sobre éste que envejece, que ya no es niño; por lo mismo, la vigilancia de los adultos se ha relajado (puede cruzar calles solo, sin supervisión/viajar solo en locomoción colectiva, vagar por su territorio y encontrarse con los pares). A su vez, la mirada de este que envejece sobre los adultos también se ha modificado. Por ejemplo, la práctica de desafiar a los adultos mediante la discusión, la crítica abierta, la puesta en duda de la necesidad de ciertas reglas, etc. aparecen con fuerza. Mediante estos recursos, el que envejece comienza a quebrar las convenciones que han reglado la relación entre adultos y niños y con ello se entronizan nuevas relaciones entre estos dos polos, anuncio del tránsito del que envejece a una nueva clase de edad: la 
juventud más temprana. Entrará a ella, además, con otra experiencia vinculada a la edad, a ser más grande, ya aludida: el placer de ejercer el poder, de mandar - hasta ese momento ejercido exclusivamente por los adultos-y de ser obedecido, la práctica del que es menor.

A partir de ese momento, la lucha del que envejece por cortar con su dependencia de los adultos es lineal: si en rigor, desde la lógica de las clases de edad en la cultura dominante, no es propio de la primera juventud, autosustentarse, ${ }^{5}$ ello no impide, en la cotidianeidad, un grado creciente de independencia de actuación por parte de los miembros de esta clase de edad respecto de los adultos en una multiplicidad de las prácticas diarias: consumo, sociabilidad, diversión, orientaciones vocacionales, vida sexual, orientaciones políticas y estéticas, cuidado y autocuidado.

Desde la posición de la clase dominante, la incorporación de los envejecientes pertenecientes a la clase de edad primera juventud al ámbito de las prácticas del trabajo en el mercado y/o de las prácticas estudiantiles de la educación superior, dinamiza todavía más esta tendencia a la autonomía de los envejecientes jóvenes respecto del mundo adulto. Se instala una nueva dinámica entre el mundo adulto y el mundo joven, la tensión de una especie de autonomía becada que el adulto debe afrontar: sustentar, financiar las necesidades de la vida práctica por la que el más joven ha optado.

La opción por la educación superior (y no por el trabajo) que el joven tenga que asumir es uno de los privilegios asignados a esta clase de edad, en conformidad con los criterios de la clase dominante. Su cumplimiento se da al interior de la familia, generando la tensión mencionada o se da en la institucionalidad educacional promovida por lo general por el Estado, a través del mecanismo de las becas. Las bases para optar a este mecanismo constituye parte de las estrategias sociales para la construcción de esta clase de edad.

La tensión en las relaciones de las clases de edad adulto/mundo juvenil que genera la obligación de los adultos de financiar la opción de los estudios superiores de uno de los segmentos de la clase de edad juventud (primera juventud), no cesará hasta el momento en que el

$5 \mathrm{Al}$ interior de esta lógica de la visión dominante, en verdad, la sustentabilidad económica de la clase de edad primera juventud, es uno de los deberes de los adultos consagrado en la legislación positiva. En la vida cotidiana opera como una especie de pacto entre clases de edad que regula los intercambios económicos entre ambas clases de edad. 
joven abandone el hogar de sus padres ya sea para fundar una nueva familia o para empoderar su propia independencia que pasa también, sin duda, por su incorporación al mercado de trabajo.

Todo ello marcará el tránsito del envejeciente a una nueva clase de edad: la adultez.

\section{d) El habitus de la adultez y de la adultez mayor}

El sujeto adulto domina a los integrantes de las otras clases de edad en el campo social de la edad. Desde los campos sociales de la economía, la educación, el mercado de trabajo, la política, la creación y la aplicación de las leyes, los adultos crean las condiciones materiales y simbólicas para legitimar y reproducir su condición de edad dominante.

En las prácticas sociales, es privilegio de la clase de edad adulta dirigir y gestionar todas y cada una de las instituciones que conforman la sociedad, desde la familia hasta la institucionalidad del estado y de mercado.

Las regulaciones etarias existentes para el retiro programado y legitimado del mercado de trabajo de los adultos — prácticas sociales jubilatorias - señalan la frontera de una nueva clase de edad: la adultez mayor.

Esta última clase de edad es el resultado de procesos históricos que han derivado en la disminución creciente de las tasas de la mortalidad adulta y de los desarrollos de los estados de bienestar europeos que determinaron la desvinculación del aparato productivo del capital monetario del segmento más envejecido de los adultos, generalizándose después como un derecho de la clase trabajadora envejecida.

En este contexto configurativo de uno de los subcampos de la edad, el de las clases de edad, los sujetos construyen sus habitus de edad y por lo mismo sus prácticas sociales individuales tenderán a conformarse dentro de estas fronteras etarias.

\section{e) El habitus generacional}

Si las clases de edad conforman un subcampo dentro del campo social de la edad, con edades distintas, históricamente generadas y con una cierta estabilidad estructural, dentro del campo social de la edad, las generaciones aparecen como el factor que puede hacer evolucionar aspectos específicos de ciertas clases de edad y a lo largo del tiempo, hasta cambiar, la relaciones y la propia identidad de las clases de edad. 
La postulación de un habitus de edad generacional se conecta con el dinamismo histórico que muestra el campo de la edad y en el que el grupo generacional emerge como un agente del campo, dispuesto a proponer apuestas nuevas, introduciendo prácticas nuevas dentro de la clase de edad de la cual se trate y renovando así los contenidos de la clase de edad respectiva, lo que no es otra cosa que el revalorar, darle un nuevo plus, al capital edad de esa clase de edad.

Es preciso acotar en este momento lo que aquí se entiende por generación. Este es un concepto profusamente usado en especial en las artes y la literatura así como en la historia de la cultura.

A diferencia de los usos que en esos contextos se hacen del vocablo generación, en el presente artículo y desde la perspectiva de la producción de las edades, se lo usará al interior de las siguientes precisiones:

i) No refiere a un grupo concreto de individuos sino a un conjunto de personas que comparten una misma situación objetiva en el espacio social.

ii) El rasgo de la contemporaneidad de lo generacional solo es relevante en la medida en que el ser contemporáneo crea la oportunidad de participación en unos mismos eventos al interior de unas condiciones de existencia parecidas.

iii) Tales condiciones de existencia - materiales y socialesademás, enmarcan las diferencias entre las generaciones, puesto que los sujetos ocupan posiciones diversas en el espacio social lo que implica efectos diferenciados sobre sus respectivos habitus.

iv) El habitus generacional, pues, no es postulable sólo al interior de las coincidencias etarias sino que a ello es necesario agregar la consideración del conjunto de las coincidencias de las condiciones sociales y materiales en las cuales se ha desarrollado un grupo de individuos determinado.

v) Ambas coincidencias - misma época, misma posición en la estructura social - permiten postular prácticas, sensibilidades, posturas, gustos, tendencialmente coincidentes, es decir habitus coincidentes, habitus generacionales.

vi) El trazado de frontera entre una generación y otra, implica, también, el conocimiento del devenir histórico de los campos sociales involucrados en la observación. En este sentido, generación y habitus generacional serán unidades postuladas de la realidad social.

vii) En otros contextos, las generaciones serán el resultado de las dinámicas políticas de producción, reproducción, sustitución de gru- 
pos. Esto es marcado en determinados campos sociales (arte, política, deporte, etc.) en los que suele hablarse (en función de los intereses de los jóvenes) de la necesidad del recambio generacional o en las dinámicas de disputa entre los «jóvenes» y los «viejos».

El habitus generacional de la edad hay que ubicarlo necesariamente al interior del contexto recién aludido de la generación.

La coexistencia en un mismo tiempo encasilla a los individuos al interior de una misma clase de edad y su habitus de edad le permitirá reconocerse en ella — «todavía estoy joven»; «ya estoy viejo», etc.—y al mismo tiempo, la posición en el espacio social, podrá hacerlo coincidir con otros, con aquellos de su misma clase social, en prácticas de edad, en sensibilidades, en visiones de la edad, la propia o la de otras clases de edad, y en percepciones sobre la sociedad y la cultura.

Es entonces la ocasión de hablar de habitus generacional de la edad.

Finalmente, es necesario reforzar la idea de operar, frente a las edades, como un producto histórico socialmente construido y no como universales intercambiables de una sociedad a otra, de una época histórica a otra.

De la rigurosidad con la cual se actúe en el entendimiento y operacionalización de las edades y de sus segmentos en cuanto productos social e históricamente producidos podrá sacar mucho provecho una disciplina social como la gerontología y las que junto con ella puedan visualizarse hoy día como integrantes del campo interdisciplinario del estudio de las edades. 


\section{REFERENCIAS BIBLIOGRÁFICAS}

BOURDIEU, PIERRE (1997): Razones prácticas. Barcelona: Anagrama.

_ (1992): El sentido práctico. Madrid: Taurus.

— (1990): «Espacio social y génesis de las clases». En Sociología y cultura. México: Grijalbo.

- (1988): La distinción. Madrid: Taurus.

— y JeAn-Claude PASSERON (1970): La reproduction. París: Minuit.

Degl'InNOCEnTI, MARTA (S/F): «Pierre Bourdieu: el capital cultural y la reproducción social». En www.unlz.edu.ar.

Estramil, NAtalia (2007): «La cognición animal». Correo del Maestro $\mathrm{N}^{\circ} 132$.

GutiÉRrez, EugENio y PATRICIO RÍos (2006): «Envejecimiento y campo de la edad: elementos sobre la pertinencia del conocimiento gerontológico». Última Década N²5. Valparaíso: Ediciones CIDPA.

GARCÍA MÉNDEZ, EMILIO (1995): Derecho de la infancia-adolescencia en América Latina: de la situación irregular a la protección integral. Bogotá: Forum-Pacis.

LOPERA, FRANCISCO (S/F): «Evolución y cognición». Revista Neuropsicología, Neuropsiquiatría y Neurociencias No27. 